\title{
Establishing IPR in Gas-Condensate Reservoir: An Alternative Approach
}

\author{
Muhammad Hanif Chachar \\ PPL Chair, Institute of Petroleum \& Natural Gas Engineering \\ Mehran University of Engineering and Technology \\ Jamshoro, Pakistan \\ mhanifchachar@gmail.com \\ Abdul Haque Tunio \\ Institute of Petroleum \& Natural Gas Engineering \\ Mehran University of Engineering and Technology \\ Jamshoro, Pakistan \\ haque.tunio@faculty.muet.edu.pk
}

\author{
Sarfraz Ahmed Jokhio \\ PPL Chair, Institute of Petroleum \& Natural Gas Engineering \\ Mehran University of Engineering and Technology \\ Jamshoro, Pakistan \\ sjokhio1@hotmail.com \\ Haris Ahmed Qureshi \\ PPL Chair, Institute of Petroleum \& Natural Gas Engineering \\ Mehran University of Engineering and Technology \\ Jamshoro, Pakistan \\ harisqureshi12.hq@gmail.com
}

\begin{abstract}
Inflow performance relationship (IPR) accuracy in the condensate reservoir is a long-standing problem in the oil industry. This paper presents a new approach to project the gas phase IPR in condensate reservoirs. IPR is estimated by Rawlins and Schellhardt equation whereas the gas pseudo-pressure function is solved by two methods and their IPRs are compared. Additionally, an average of both IPR's is estimated and compared. At the reservoir pressure, the difference between both flow rates is negligible i.e. at 6750 psi, the flow rate difference is $0.55 \mathrm{MMSCF} / \mathrm{D}$. As pressure declines the difference is increasing at one stage, it is observed approximately $15 \mathrm{MMSCF} / \mathrm{D}$.
\end{abstract}

Keywords-gas; condensate; condensate reservoir; Pseudopressure; Well productivity; Relative Permeability; Permeability; IPR

\section{INTRODUCTION}

The gas condensate reservoirs are difficult to predict due to its multiphase behavior. Petroleum industry is struggling to obtain the accurate Inflow Performance Relation (IPR) in condensate reservoirs for decades. There is not much research conducted in the case of calculating gas phase IPR in a condensate reservoir. It is possible to calculate IPR by using gas phase pseudo-pressure function without using the relative permeability data. Pseudo-pressure equation is solved using effective permeability data which can be obtained by pressure buildup test [1]. In the solution gas drive reservoir, two phase flow causes the curvature in IPR due to the reduction in the relative permeability of the oil phase with the depletion [2]. Two phase pseudo-steady state equation was solved [3] based on Weller's approximation of constant gas oil ratio (GOR) and constant de-saturation [4]. In this study, the proposed approach is compared with the conventional method. To generate the IPR, the relative equation in [5] is preferred. In several case studies, it has been shown through production data and well test data that condensate blockage may reduce the production from two to four times. The major cause of production loss is condensate blockage near the wellbore [6]. To understand the condensate behavior, it is divided into three regions. Region-1 (near wellbore), where both phases are presented and mobile, Region-2 where both phases are present but only gas phase is mobile, and Region-3 (above dew point pressure) where only the gas phase is present and mobile [7]. Experimental work on the long core of sandstone formation outcome shows that the mobility is increased with capillary number near the wellbore region [8]. Authors in [9] verified the existence of the three regions by using a compositional simulator. The estimation of total well productivity in condensate reservoir is complicated. Condensate extent must be known to identify the blockage effect so that remedial action can be taken [10]. In [11], common problems associated with the condensate reservoirs were investigated and several proposed solutions were reviewed. This paper provides key improvements in the calculation of gas phase pseudo-pressure function using the integral effective permeability technique proposed in [12].

\section{MODEL DESCRIPTION AND WORKING EQUATIONS}

\section{A. Gas Pseudo-Pressure Function}

It completely depends on the pressure. To calculate real gas pseudopressure function linearly, Kirchhoff integral transformation is used as follows [13]:

$$
m(p)=2 \int_{P_{w f}}^{P} \frac{p}{\mu Z} d p
$$

where $P$ : pressure, $\mu$ : viscosity, and $Z$ : compressibility factor. Authors in [7] modified and introduced the pseudopressure equation in form of three regions [7]. The total gas pseudopressure equation is:

$$
m(p)=\int_{P_{w f}}^{P_{r}}\left(\frac{k_{r o}}{\mu_{o} B_{o}} R_{s}+\frac{k_{r g}}{\mu_{g} B_{g}}\right) d p
$$

where $k_{r o}$ : relative permeability of oil, $\mu_{o}$ : viscosity of oil, $B_{o}$ : oil formation volume factor, $R s$ : the solution gas-oil ratio, and 
g: gas. Authors in [12] modified the pseudopressure equation to calculate it with the single-phase effective permeability of either gas or oil by using well test data. They proposed the following equation, using gas PVT properties:

$$
m(P)_{g}=\left[\int_{P_{w f}}^{P_{r}}\left(\frac{k \cdot k_{r g}}{B_{g} \mu_{g}}\right) \frac{R_{p}\left(1-R_{o} R_{S}\right)}{R_{p}-R_{S}}(P) d p\right]
$$

where $R p$ : producing gas-oil ratio and $R o$ : oil vapors in gas. Calculations of the classic and the proposed method are given in Table VI and VII respectively.

\section{B. Derivative of Gas Pseudo-Pressure Function}

Time dependent derivative of the pseudopressure function is required in order to obtain the integral of effective permeability. To calculate the derivative, it is necessary to obtain a pseudopressure equation. At first, the pseudopressure equation was calculated by ignoring the permeability data. Then, the derivative of pseudopressure equation is calculated.

\section{Integral of Effective Permeability}

The effective permeability data were calculated from the semi log straight line (SSL) from Shut-in versus time in Figure 1. The start of SSL shows the MTR (Middle Time Region) on PBU (Pressure Build-up) plot. The following equation is used to calculate the integral of effective permeability:

$$
\int_{P_{w f}}^{P}\left(k_{e g}\right) d p=\int_{P_{w f}}^{P}\left(k \cdot k_{r g}\right) d p=162.6\left(\frac{q_{g, \text { meas }}}{h}\right) \frac{1}{\Delta m(p)^{\prime}}
$$

where $k_{e g}$ : gas effective permeability, $q_{g \text {,meas }}$ : flow rate of gas measured, $h$ : bed thickness and $\Delta m(p)^{\prime}:$ change in pseudopressure derivative. Now, extrapolate the integral of effective permeability versus pressure to zero using curve fit software. The equation from curve is used to calculate its integral on the desired pressure (Figure 4).

\section{Effective Permeability}

Once the integral of effective permeability is calculated, we take its derivative using a two-point numerical derivative as a function of pressure:

$$
k_{e g}=\frac{\left(\int k_{e g}\right)_{2}-\left(\int k_{e g}\right)_{1}}{\left(P_{2}-P_{1}\right)}
$$

This effective permeability is used to calculate the final pseudopressure function as shown in Table VII.

\section{E. Gas Flow Rate for IPR}

To establish gas phase IPR, the equation from [5] is used. Additionally, both gas pseudopressure equations are solved and their IPRs are plotted and compared. Figures 6 and 8 show the classic and the proposed IPR whereas Figure 9 shows the comparison of both IPRs with their average.

$$
q_{g}=C \times\left[\left(m(p)_{g}\right)_{p i}^{2}-\left(m(p)_{g}\right)_{p w f}^{2}\right]^{n}
$$

where $C$ : flow coefficient, $n$ : deliverability exponent, $m(p)_{g}$ : gas pseudopressure function, $p i$ : initial pressure and $p_{w f}$ : wellbore flowing pressure.

\section{STEP-BY-STEP PROCEDURE TO GENERATE IPR}

This method of calculating IPR is slightly different from the classic one. Its steps are:

1. Calculate gas properties (viscosity, density, compressibility) by conventional equations.

2. Convert well test data into gas pseudopressure function, ignoring permeability terms.

3. Calculate the derivative of the time $\log \left(\frac{d \Delta m(p)}{d \ln (t)}\right)$ of pseudopressure data.

4. Plot well test data pressure versus time on semi-log and find the straight-line.

5. Estimate the integral of effective permeability following the straight line from the previous step.

6. Plot the estimated integral of effective permeability versus pressure extrapolated to zero limits. To get good curve fit the equation's both limits should be extrapolated to zero.

7. Calculate the integral of effective permeability values using the generated equation from the curve obtained in Step 6.

8. Calculate the effective permeability using a two-point numerical derivative as a function of pressure from the previous step.

9. Calculate the pseudopressure function again this time including the effective permeability data.

10. Finally, establish condensate well performance by using the equation from [5].

\section{CONCLUDING INTERPRETATION}

IPR is often used to predict the natural flow of the well so it should be properly selected. This study presents a new approach to estimate IPR in a gas condensate reservoir. Furthermore, it concludes with the following observations:

- A method is proposed to calculate pseudopressure equation for plotting IPR in gas condensate reservoir. It does not require relative permeability data, as effective permeability can be easily calculated from its integral.

- Effective permeability must be used before solving the pseudopressure integral. The comparison of IPR with the classic method shows that as pressure declines the flow rate difference in classic method and the proposed one increases. It can reduce the error by 1-15MMSCF/d.

\section{RECOMMENDATIONS}

IPR can obtain optimum flow of the well. It is highly recommended to use the proposed method to reduce errors in IPR calculations. The error percentage shows the reliability of this work. For additional research, three-phase reservoir should be considered, and optimum flow can be obtained by plotting TPR vs. IPR

\section{A. Abbreviations and Acronyms}

$\mathrm{PPF}=$ Pseudopressure function

$\mathrm{GOR}=$ Gas Oil Ratio

SSL $=$ Semi-log-straight-line

R-1 = Region-1 
$\mathrm{R}-2$ = Region-2

R-3 = Region-3

$B_{g} \quad=$ Gas formation volume factor

$\mu_{g} \quad=$ Gas viscosity

$K_{e g}=$ Gas effective-permeability

$K_{r g}=$ Gas relative-permeability

$q_{g}=$ Gas flow-rate

$q_{o} \quad=$ Oil flow-rate

$R_{p} \quad$ = Producing gas-oil ratio

$R_{s o}=$ Solution gas-oil ratio

$m(p)=$ Pseudo-pressure function

$m(p)^{\prime}=$ Derivative of pseudo-pressure function

$\Delta m(p)=$ Change in pseudo-pressure function

B. Figures and Tables

TABLE I. RESERVOIR AND FLUID DATA [14]

\begin{tabular}{|c|c|c|c|c|c|}
\hline Symbol & Value & Unit & Symbol & Value & Unit \\
\hline $\mathrm{Pi}$ & 6750 & Pisa & $\mathrm{qg}$ & 75.4 & $\mathrm{MSCF} / \mathrm{D}$ \\
\hline $\mathrm{Pd}$ & 6750 & Pisa & $\mathrm{qo}$ & 2.8 & $\mathrm{STB} / \mathrm{D}$ \\
\hline $\mathrm{Rp} / \mathrm{GOR}$ & 10417 & $\mathrm{SCF} / \mathrm{STB}$ & $\mathrm{h}$ & 216.5 & $\mathrm{ft}$ \\
\hline $\mathrm{T}$ & 814 & ${ }^{\circ} \mathrm{R}$ & $\Phi$ & 0.062 & \\
\hline $\mathrm{Gas} \mathrm{SG}$ & 0.94 & & $\mathrm{rw}$ & 0.54 & $\mathrm{ft}$ \\
\hline $\mathrm{MW}$ & 27.17 & & $\mathrm{API}$ & 50 & {$[$ Assumed] } \\
\hline$\Delta \mathrm{T}$ & 2.85 & ${ }^{\circ} \mathrm{F} / 100 \mathrm{ft}$ & & & \\
\hline
\end{tabular}

TABLE II. WELL TEST DATA [14]

\begin{tabular}{|c|c|c|c|c|c|}
\hline Time (hrs) & $\boldsymbol{P}$ (psi) & Time (hrs) & $\boldsymbol{P}$ (psi) & Time (hrs) & $\boldsymbol{P}$ (psi) \\
\hline 0 & 1083.1 & 6 & 2759.4 & 50 & 6487.3 \\
\hline 0.167 & 1174.5 & 8 & 3246.5 & 58 & 6507.6 \\
\hline 0.333 & 1226.7 & 12 & 4210 & 68 & 6526.5 \\
\hline 0.5 & 1303.6 & 16 & 5162 & 82 & 6556.9 \\
\hline 1 & 1490.6 & 22 & 6161 & 97 & 6574.3 \\
\hline 2 & 1751.6 & 28 & 6336.5 & 112 & 6587.3 \\
\hline 3 & 2046 & 34 & 6406.1 & 141 & 6601.8 \\
\hline 4 & 2279.4 & 42 & 6452.5 & $\operatorname{Pr}$ & 6750 \\
\hline
\end{tabular}

TABLE III. GAS PVT PROPERTIES AT WELL TEST PRESSURE

\begin{tabular}{|c|c|c|c|c|c|c|}
\hline $\begin{array}{c}\boldsymbol{P} \\
\mathbf{p s i}\end{array}$ & $\boldsymbol{Z}$ & $\begin{array}{c}\boldsymbol{B}_{\boldsymbol{g}} \\
\mathbf{b b l} / \mathbf{S C F}\end{array}$ & $\begin{array}{c}\boldsymbol{\rho}_{\boldsymbol{g}} \\
\mathbf{g m} / \mathbf{c c}\end{array}$ & $\begin{array}{c}\mu_{\boldsymbol{g}} \\
\mathbf{c c}\end{array}$ & $\begin{array}{c}\boldsymbol{R}_{\boldsymbol{s}} \\
\text { SCF/STB }\end{array}$ & $\begin{array}{c}\boldsymbol{R}_{\boldsymbol{o}} \\
\text { STB/SCF }\end{array}$ \\
\hline 1083.1 & 0.9145 & 0.0034 & 0.0539 & 0.0080 & 297.945 & $1.4 \mathrm{E}-05$ \\
\hline 1174.5 & 0.9101 & 0.0031 & 0.0585 & 0.0082 & 327.131 & $1.55 \mathrm{E}-05$ \\
\hline 1226.7 & 0.9075 & 0.0030 & 0.0611 & 0.0083 & 343.959 & $1.63 \mathrm{E}-05$ \\
\hline 1303.6 & 0.9038 & 0.0028 & 0.0649 & 0.0085 & 368.948 & $1.75 \mathrm{E}-05$ \\
\hline 1490.6 & 0.8947 & 0.0024 & 0.0742 & 0.0090 & 430.644 & $2.04 \mathrm{E}-05$ \\
\hline 1751.6 & 0.8820 & 0.0020 & 0.0872 & 0.0097 & 518.739 & $2.41 \mathrm{E}-05$ \\
\hline 2046.0 & 0.8672 & 0.0017 & 0.1019 & 0.0105 & 620.549 & $2.82 \mathrm{E}-05$ \\
\hline 2279.4 & 0.8716 & 0.0015 & 0.1135 & 0.0113 & 702.899 & $3.14 \mathrm{E}-05$ \\
\hline 2759.4 & 0.8807 & 0.0013 & 0.1374 & 0.0130 & 876.247 & $3.81 \mathrm{E}-05$ \\
\hline 3246.5 & 0.8899 & 0.0011 & 0.1617 & 0.0151 & 1056.97 & $4.54 \mathrm{E}-05$ \\
\hline 4210 & 0.9157 & 0.0008 & 0.2097 & 0.0200 & 1426.45 & $6.22 \mathrm{E}-05$ \\
\hline 5162.0 & 0.9962 & 0.0007 & 0.2572 & 0.0273 & 1804.60 & $8.39 \mathrm{E}-05$ \\
\hline 6161.0 & 1.0806 & 0.0007 & 0.3069 & 0.0376 & 2213.14 & 0.000115 \\
\hline 6336.5 & 1.0954 & 0.0007 & 0.3157 & 0.0397 & 2286.02 & 0.000121 \\
\hline 6406.1 & 1.1013 & 0.0007 & 0.3192 & 0.0406 & 2315.01 & 0.000124 \\
\hline 6452.5 & 1.1052 & 0.0007 & 0.3215 & 0.0413 & 2334.36 & 0.00012 \\
\hline 6487.3 & 1.1082 & 0.0007 & 0.3232 & 0.0417 & 2348.89 & 0.00012 \\
\hline 6507.6 & 1.1099 & 0.0007 & 0.3242 & 0.0420 & 2357.37 & 0.000128 \\
\hline 6526.5 & 1.1115 & 0.0006 & 0.3252 & 0.0422 & 2365.27 & 0.000129 \\
\hline 6556.9 & 1.1140 & 0.0006 & 0.3267 & 0.0427 & 2377.98 & 0.000130 \\
\hline 6574.3 & 1.1155 & 0.0006 & 0.3275 & 0.0429 & 2385.26 & 0.000131 \\
\hline 6587.3 & 1.1166 & 0.0006 & 0.3282 & 0.0431 & 2390.70 & 0.000131 \\
\hline 6601.8 & 1.1178 & 0.0006 & 0.3289 & 0.0433 & 2396.77 & 0.000132 \\
\hline 6750.0 & 1.1304 & 0.0006 & 0.3363 & 0.0454 & 2458.94 & 0.000138 \\
\hline
\end{tabular}

TABLE IV. PPF, ITS DERIV ATIVE AND INTEGRAL OF EFFECTIVE PERMEABILITY

\begin{tabular}{|c|c|c|c|}
\hline $\begin{array}{c}P \\
\text { psi }\end{array}$ & $\begin{array}{c}\Delta m(p) \\
\mathrm{MMpsi}^{2} / \mathbf{c p}\end{array}$ & $\Delta m(p)^{\prime}$ & $\int K e g$ \\
\hline 1083.1 & 19.87139 & & \\
\hline 1174.5 & 23.33366 & & \\
\hline 1226.7 & 25.40711 & & \\
\hline 1303.6 & 28.58367 & 7.475785 & \\
\hline 1490.6 & 36.87831 & 11.75797 & \\
\hline 1751.6 & 49.68891 & 29.24621 & \\
\hline 2046 & 65.64563 & 38.57398 & \\
\hline 2279.4 & 79.14166 & 55.36694 & \\
\hline 2759.4 & 108.0837 & 75.90609 & \\
\hline 3246.5 & 138.2613 & 107.2502 & \\
\hline 4210 & 196.2917 & 139.7734 & \\
\hline 5162 & 246.5346 & 135.301 & \\
\hline 6161 & 287.666 & 93.06389 & SSL \\
\hline 6336.5 & 293.6161 & 56.13135 & 0.001008854 \\
\hline 6406.1 & 295.8697 & 15.22777 & 0.003718756 \\
\hline 6452.5 & 297.3387 & 8.76812 & 0.006458438 \\
\hline 6487.3 & 298.423 & 5.343989 & 0.010596647 \\
\hline 6507.6 & 299.0485 & 4.866248 & 0.011636966 \\
\hline 6526.5 & 299.6263 & 4.524248 & 0.012516635 \\
\hline 6556.9 & 300.5464 & 3.375618 & 0.016775701 \\
\hline 6574.3 & 301.0679 & 3.658003 & 0.015480678 \\
\hline 6587.3 & 301.4551 & 2.58145 & 0.021936645 \\
\hline 6601.8 & 301.8845 & & \\
\hline 6750 & 306.1246 & & \\
\hline
\end{tabular}

TABLE V. PVT PROPERTIES AT ASSUMED PRESSURE

\begin{tabular}{|c|c|c|c|c|c|c|}
\hline $\begin{array}{c}\boldsymbol{P} \\
\mathbf{p s i}\end{array}$ & $\boldsymbol{Z}$ & $\begin{array}{c}\boldsymbol{B}_{\boldsymbol{g}} \\
\mathbf{b b l / S C F}\end{array}$ & $\begin{array}{c}\boldsymbol{\rho}_{\boldsymbol{g}} \\
\mathbf{g m} / \mathbf{c c}\end{array}$ & $\begin{array}{c}\mu_{\boldsymbol{g}} \\
\mathbf{c c}\end{array}$ & $\begin{array}{c}\boldsymbol{R}_{\boldsymbol{s}} \\
\text { SCF/STB }\end{array}$ & $\begin{array}{c}\boldsymbol{R}_{\boldsymbol{o}} \\
\text { STB/SCF }\end{array}$ \\
\hline 100 & 0.98995 & 0.04061 & 0.0049 & 0.0062 & 19.08302 & $1.4 \mathrm{E}-05$ \\
\hline 300 & 0.97359 & 0.01331 & 0.0149 & 0.0065 & 67.76515 & $3.4 \mathrm{E}-06$ \\
\hline 600 & 0.94906 & 0.00648 & 0.0298 & 0.0070 & 150.7455 & $4.83 \mathrm{E}-06$ \\
\hline 900 & 0.92348 & 0.00421 & 0.0448 & 0.0076 & 240.6389 & $1.09 \mathrm{E}-05$ \\
\hline 1200 & 0.90888 & 0.00310 & 0.0597 & 0.0083 & 335.3379 & $1.59 \mathrm{E}-05$ \\
\hline 1500 & 0.89428 & 0.00244 & 0.0747 & 0.0090 & 433.7788 & $2.05 \mathrm{E}-05$ \\
\hline 1800 & 0.87969 & 0.00200 & 0.0896 & 0.0098 & 535.3081 & $2.48 \mathrm{E}-05$ \\
\hline 2100 & 0.86826 & 0.00169 & 0.1046 & 0.0107 & 639.48 & $2.89 \mathrm{E}-05$ \\
\hline 2400 & 0.87393 & 0.00149 & 0.1195 & 0.0117 & 745.9688 & $3.31 \mathrm{E}-05$ \\
\hline 2700 & 0.87962 & 0.00133 & 0.1345 & 0.0128 & 854.5257 & $3.73 \mathrm{E}-05$ \\
\hline 3000 & 0.88530 & 0.00121 & 0.1494 & 0.0140 & 964.9535 & $4.16 \mathrm{E}-05$ \\
\hline 3300 & 0.89099 & 0.00110 & 0.1644 & 0.0153 & 1077.092 & $4.62 \mathrm{E}-05$ \\
\hline 3600 & 0.89668 & 0.00102 & 0.1793 & 0.0168 & 1190.809 & $5.11 \mathrm{E}-05$ \\
\hline 3900 & 0.88955 & 0.00093 & 0.1943 & 0.0184 & 1305.991 & $5.63 \mathrm{E}-05$ \\
\hline 4200 & 0.91490 & 0.00089 & 0.2092 & 0.0202 & 1422.542 & $6.2 \mathrm{E}-05$ \\
\hline 4500 & 0.94025 & 0.00085 & 0.2242 & 0.0222 & 1540.379 & $6.82 \mathrm{E}-05$ \\
\hline 4800 & 0.96561 & 0.00082 & 0.2391 & 0.0244 & 1659.429 & $7.49 \mathrm{E}-05$ \\
\hline 5100 & 0.99096 & 0.00079 & 0.2541 & 0.0268 & 1779.628 & $8.23 \mathrm{E}-05$ \\
\hline 5400 & 1.01632 & 0.00077 & 0.2690 & 0.0295 & 1900.917 & $9.05 \mathrm{E}-05$ \\
\hline 5700 & 1.04167 & 0.00075 & 0.2840 & 0.0324 & 2023.246 & $9.94 \mathrm{E}-05$ \\
\hline 6000 & 1.06702 & 0.00073 & 0.2989 & 0.0357 & 2146.567 & 0.000109 \\
\hline 6300 & 1.09238 & 0.00071 & 0.3139 & 0.0393 & 2270.839 & 0.00012 \\
\hline 6750 & 1.13041 & 0.00068 & 0.3363 & 0.0454 & 2458.946 & 0.000138 \\
\hline
\end{tabular}

The curve in Figure 4 is obtained from the equation generated by the curve fit. The smooth curve is an indication of accuracy and it should be used to calculate the other required data. The curve in Figure 6 shows the trend of effective permeability of gas against pressure. As the pressure is increased, the effective permeability of the gas is also increased. The graph trend in Figure 9 shows that error is reduced by $1-15 \mathrm{MMSCF} / \mathrm{d}$. The difference between average IPR and classic IPR is $0.5-7.5 \mathrm{MSCF} / \mathrm{d}$. 
TABLE VI. FINAL PPF AND FLOW RATE - CLASSIC METHOD

\begin{tabular}{|c|c|c|c|c|}
\hline $\begin{array}{c}\boldsymbol{P} \\
\mathbf{p s i}\end{array}$ & $\begin{array}{c}\boldsymbol{m}(\boldsymbol{p})_{\boldsymbol{g}} \\
\mathbf{M M p s i}^{\mathbf{c}} / \mathbf{c p}\end{array}$ & $\int \boldsymbol{K}_{\boldsymbol{e g}}$ & $\begin{array}{c}\boldsymbol{m}(\boldsymbol{p})_{\boldsymbol{g}} \text { (Final) } \\
\mathbf{M M S C F}\end{array}$ & $\begin{array}{c}\boldsymbol{q}_{\boldsymbol{g}} \\
\mathbf{M M S C F} / \mathbf{D}\end{array}$ \\
\hline 100 & 0.196978075 & $6.50266 \mathrm{E}-05$ & $1.28088 \mathrm{E}-05$ & 94.05954 \\
\hline 300 & 1.742696934 & $8.13784 \mathrm{E}-05$ & 0.00014181 & 94.04973 \\
\hline 600 & 6.778583051 & 0.000100002 & 0.00067787 & 94.009 \\
\hline 900 & 14.82858679 & 0.000117101 & 0.001736445 & 93.92854 \\
\hline 1200 & 25.52693376 & 0.000134243 & 0.003426806 & 93.80002 \\
\hline 1500 & 38.4846738 & 0.000152154 & 0.005855598 & 93.61529 \\
\hline 1800 & 53.3722675 & 0.000171361 & 0.009145922 & 93.36488 \\
\hline 2100 & 69.85112141 & 0.000192357 & 0.013436386 & 93.03811 \\
\hline 2400 & 87.42029189 & 0.000215685 & 0.018855232 & 92.62498 \\
\hline 2700 & 105.6132162 & 0.000241992 & 0.025557541 & 92.11336 \\
\hline 3000 & 124.1582217 & 0.000272102 & 0.03378372 & 91.48445 \\
\hline 3300 & 142.8181289 & 0.000307105 & 0.043860203 & 90.71261 \\
\hline 3600 & 161.3865833 & 0.000348495 & 0.056242486 & 89.76188 \\
\hline 3900 & 179.8151745 & 0.000398395 & 0.071637542 & 88.57631 \\
\hline 4200 & 197.7524845 & 0.000459941 & 0.090954501 & 87.08307 \\
\hline 4500 & 214.7604013 & 0.000537981 & 0.11553694 & 85.17347 \\
\hline 4800 & 230.77076 & 0.00064044 & 0.147794924 & 82.65125 \\
\hline 5100 & 245.7339194 & 0.000781243 & 0.191977821 & 79.16496 \\
\hline 5400 & 259.6153259 & 0.000987312 & 0.256321205 & 74.01772 \\
\hline 5700 & 272.3926848 & 0.001318394 & 0.359120865 & 65.6006 \\
\hline 6000 & 284.0536412 & 0.001939033 & 0.550789526 & 49.10788 \\
\hline 6300 & 294.5938878 & 0.003527854 & 0.811762171 & 27.12217 \\
\hline 6750 & 308.3105787 & 0.003211887 & 0.990258889 & 0 \\
\hline
\end{tabular}

TABLE VII. PPF AND FLOW RATE - PROPOSED METHOD

\begin{tabular}{|c|c|c|c|}
\hline $\boldsymbol{P}(\mathbf{p s i})$ & $\boldsymbol{k}_{\boldsymbol{e g}}$ & $\boldsymbol{m}(\boldsymbol{p})_{\boldsymbol{g}}\left(\mathbf{M M p s i}{ }^{2} / \mathbf{c p}\right)$ & $\boldsymbol{q}_{\boldsymbol{g}}(\mathbf{M M S C F} / \mathbf{D})$ \\
\hline 100 & 0 & $3.9 \mathrm{E}+08$ & 80.29606 \\
\hline 300 & $8.17589 \mathrm{E}-08$ & $1.41 \mathrm{E}+08$ & 80.40645 \\
\hline 600 & $6.2078 \mathrm{E}-08$ & $4.88 \mathrm{E}+08$ & 80.25238 \\
\hline 900 & $5.69979 \mathrm{E}-08$ & $9.64 \mathrm{E}+08$ & 80.04083 \\
\hline 1200 & $5.71385 \mathrm{E}-08$ & $1.57 \mathrm{E}+09$ & 79.76904 \\
\hline 1500 & $5.97042 \mathrm{E}-08$ & $2.33 \mathrm{E}+09$ & 79.43118 \\
\hline 1800 & $6.4023 \mathrm{E}-08$ & $3.26 \mathrm{E}+09$ & 79.01951 \\
\hline 2100 & $6.99885 \mathrm{E}-08$ & $4.36 \mathrm{E}+09$ & 78.52535 \\
\hline 2400 & $7.77579 \mathrm{E}-08$ & $5.66 \mathrm{E}+09$ & 77.94393 \\
\hline 2700 & $8.76901 \mathrm{E}-08$ & $7.17 \mathrm{E}+09$ & 77.26862 \\
\hline 3000 & $1.00368 \mathrm{E}-07$ & $8.91 \mathrm{E}+09$ & 76.48462 \\
\hline 3300 & $1.16677 \mathrm{E}-07$ & $1.09 \mathrm{E}+10$ & 75.572 \\
\hline 3600 & $1.37967 \mathrm{E}-07$ & $1.33 \mathrm{E}+10$ & 74.50349 \\
\hline 3900 & $1.66333 \mathrm{E}-07$ & $1.61 \mathrm{E}+10$ & 73.23101 \\
\hline 4200 & $2.05152 \mathrm{E}-07$ & $1.94 \mathrm{E}+10$ & 71.71536 \\
\hline 4500 & $2.60132 \mathrm{E}-07$ & $2.34 \mathrm{E}+10$ & 69.90629 \\
\hline 4800 & $3.41533 \mathrm{E}-07$ & $2.82 \mathrm{E}+10$ & 67.69041 \\
\hline 5100 & $4.69341 \mathrm{E}-07$ & $3.42 \mathrm{E}+10$ & 64.87688 \\
\hline 5400 & $6.86896 \mathrm{E}-07$ & $4.22 \mathrm{E}+10$ & 61.11419 \\
\hline 5700 & $1.10361 \mathrm{E}-06$ & $5.35 \mathrm{E}+10$ & 55.66273 \\
\hline 6000 & $2.0688 \mathrm{E}-06$ & $7.17 \mathrm{E}+10$ & 46.58986 \\
\hline 6300 & $5.29607 \mathrm{E}-06$ & $1.1 \mathrm{E}+11$ & 25.98219 \\
\hline 6750 & $7.0215 \mathrm{E}-07$ & $1.45 \mathrm{E}+11$ & 0 \\
\hline & & & \\
\hline
\end{tabular}

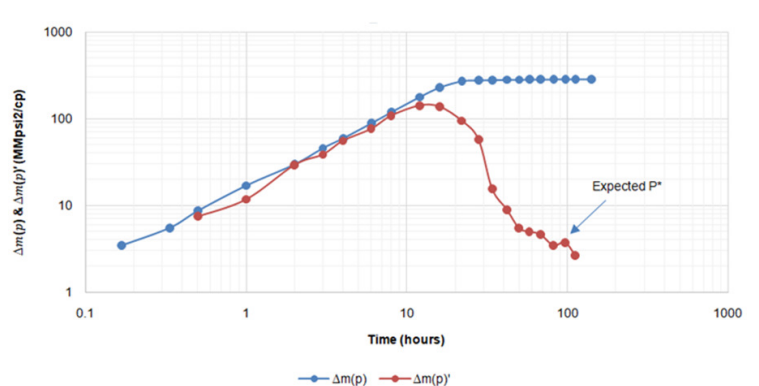

Fig. 1. Pseudopressure function and its derivative vs. time

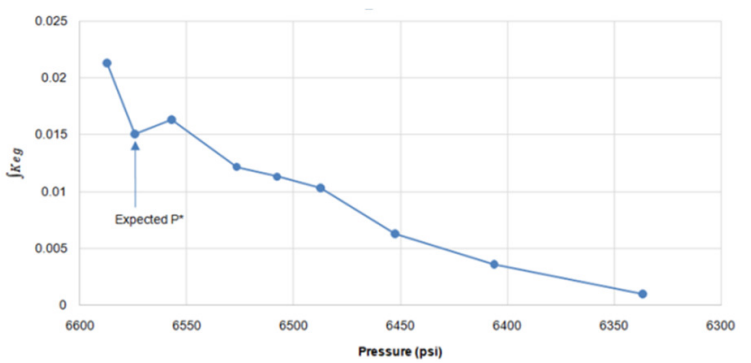

Fig. 2. Integral of gas effective permeability as a function of pressure

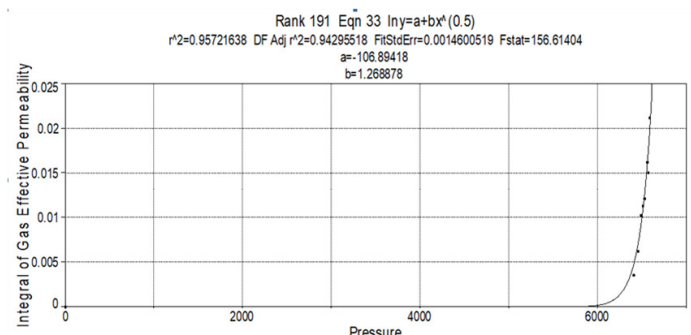

Fig. 3. Integral of gas effective permeability as a function of pressure extrapolated to zero

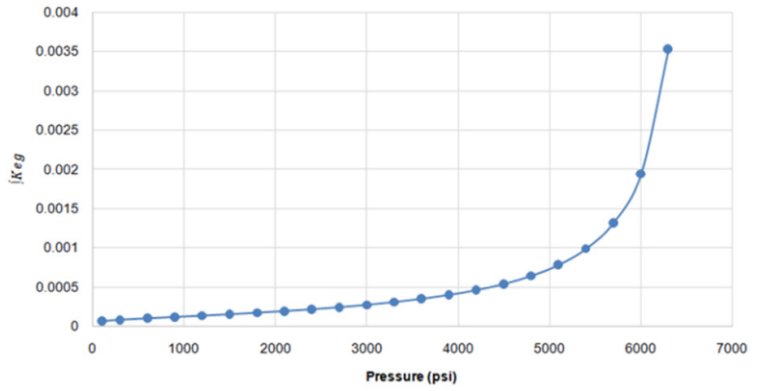

Fig. 4. Integral of effective permeability versus pressure at assumed pressure points

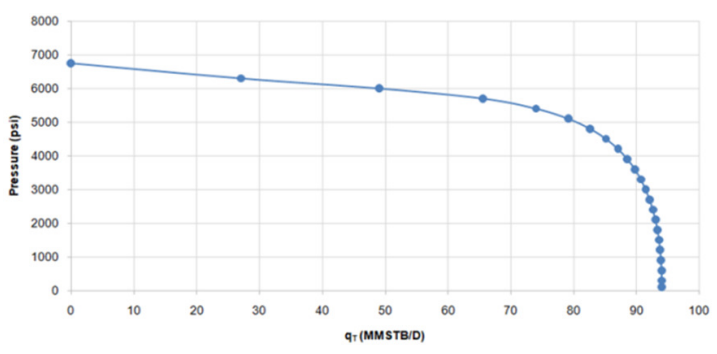

Fig. 5. IPR via classic method

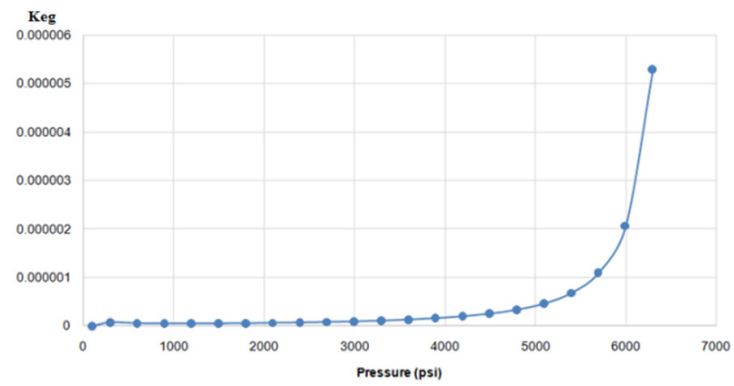

Fig. 6. Gas effective permeability vs. pressure 
TABLE VIII. PROPOSED AND CLASSIC METHOD COMPARISON

\begin{tabular}{|c|c|c|c|c|}
\hline $\begin{array}{c}\boldsymbol{P} \\
\text { (psi) }\end{array}$ & $\begin{array}{c}\boldsymbol{q}_{\boldsymbol{g}} \\
\text { (MMSCF/D) }\end{array}$ & $\begin{array}{c}\boldsymbol{q}_{\boldsymbol{g}} \\
\text { (MMSCF/D) }\end{array}$ & $\begin{array}{c}\boldsymbol{q}_{\boldsymbol{g}} \text { (difference) } \\
\text { (MMSCF/D) }\end{array}$ & $\begin{array}{c}\boldsymbol{q}_{\boldsymbol{g}} \text { (avg) } \\
\text { (MMSCF/D) }\end{array}$ \\
\hline 100 & 94.05954 & 80.29606 & 13.76348 & 87.1778 \\
\hline 300 & 94.04973 & 80.40645 & 13.64328 & 87.22809 \\
\hline 600 & 94.009 & 80.25238 & 13.75662 & 87.13069 \\
\hline 900 & 93.92854 & 80.04083 & 13.88771 & 86.98468 \\
\hline 1200 & 93.80002 & 79.76904 & 14.03098 & 86.78453 \\
\hline 1500 & 93.61529 & 79.43118 & 14.18411 & 86.52323 \\
\hline 1800 & 93.36488 & 79.01951 & 14.34537 & 86.1922 \\
\hline 2100 & 93.03811 & 78.52535 & 14.51276 & 85.78173 \\
\hline 2400 & 92.62498 & 77.94393 & 14.68105 & 85.28445 \\
\hline 2700 & 92.11336 & 77.26862 & 14.84475 & 84.69099 \\
\hline 3000 & 91.48445 & 76.48462 & 14.99984 & 83.98453 \\
\hline 3300 & 90.71261 & 75.572 & 15.14061 & 83.1423 \\
\hline 3600 & 89.76188 & 74.50349 & 15.25839 & 82.13268 \\
\hline 3900 & 88.57631 & 73.23101 & 15.3453 & 80.90366 \\
\hline 4200 & 87.08307 & 71.71536 & 15.36771 & 79.39921 \\
\hline 4500 & 85.17347 & 69.90629 & 15.26718 & 77.53988 \\
\hline 4800 & 82.65125 & 67.69041 & 14.96084 & 75.17083 \\
\hline 5100 & 79.16496 & 64.87688 & 14.28808 & 72.02092 \\
\hline 5400 & 74.01772 & 61.11419 & 12.90353 & 67.56595 \\
\hline 5700 & 65.6006 & 55.66273 & 9.937865 & 60.63167 \\
\hline 6000 & 49.10788 & 46.58986 & 2.518028 & 47.84887 \\
\hline 6300 & 27.12217 & 25.98219 & 1.139977 & 26.55218 \\
\hline 6750 & 0 & 0 & & 0 \\
\hline
\end{tabular}

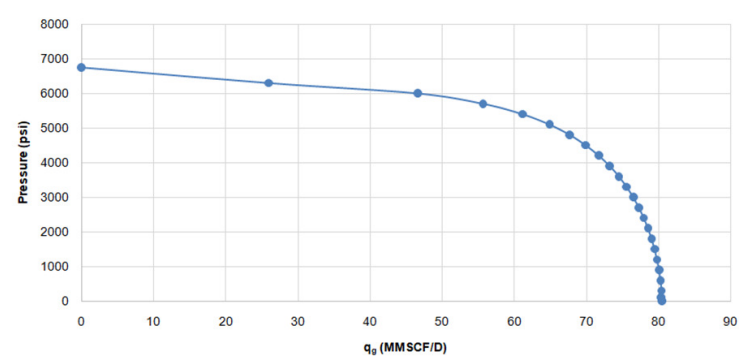

Fig. 7. IPR via the proposed method

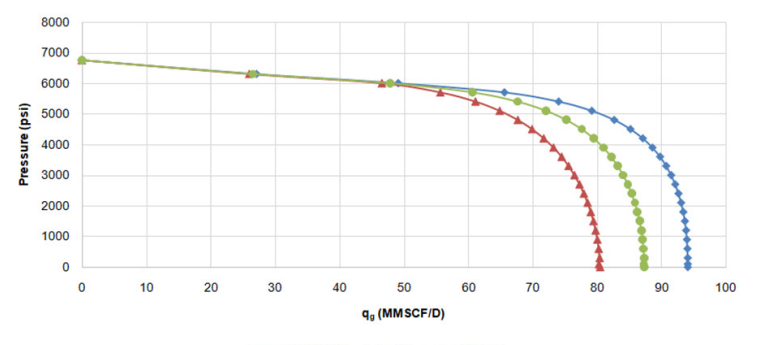

Fig. 8. IPRs via classic and proposed method and their average

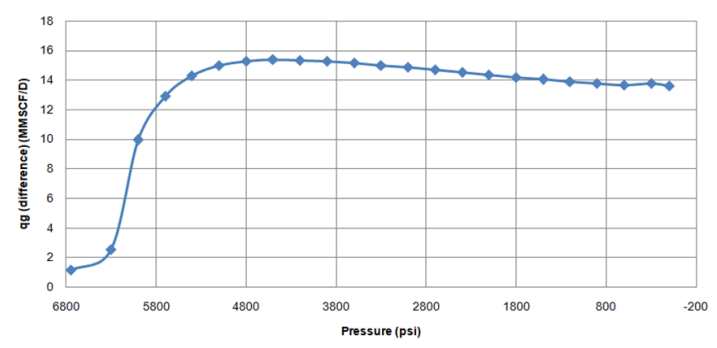

Fig. 9. Variation in gas flow rate versus pressure

\section{ACKNOWLEDGMENT}

The authors are grateful to Pakistan Petroleum Ltd, and the Institute of Petroleum \& Natural Gas Engineering, Mehran University of Engineering \& Technology, Jamshoro.

\section{REFERENCES}

[1] S. A. Jokhio, D. Tiab, "Establishing inflow performance relationship (IPR) for gas condensate wells", SPE Gas Technology Symposium, Calgary, USA, 30 April-2 May, 2002

[2] H. H. Evinger, M. Muskat, "Calculation of theoretical productivity factors", Transactions of the AIME, Vol. 146, No. 1, pp. 126-139, 1942

[3] J. T. Vogel, "Inflow Performance Relationships for solution-gas drive wells", Journal of Petroleum Technology, Vol. 20, No. 1, pp. 83-92, 1968

[4] W. T. Weller, "Reservoir performance during two phase flow", Journal of Petroleum Technology, Vol. 18, No. 2, pp. 240-245, 1966

[5] E. L. Rawlins, M. A. Schellhardt, Backpressure data on natural gas wells and their application to production practices, Literary Licensing, 1935

[6] D. Afidick, N. J. Kaczorowski, S. Bette, "Production performance of a retrograde gas reservoir: a case study of the Arun field", SPE Asia Pacific Oil and Gas Conference, Melbourne, Australia, November 7-10, 1994

[7] O. Fevang, C. H. Whitson, "Modeling gas condensate deliverability", SPE Reservoir Engineering, Vol. 11, No. 4, pp. 221-230, 1995

[8] R. Mott, A. Cable, M. Spearing, "A new method of measuring relative permeabilities for calculating gas-condensate well deliverability", 1999 SPE Annual Technical Conference and Exhibition, Houston, USA, October 3-6, 1999

[9] A. C. Gringarten, A. Al-Lamki, S. Daungkaew, R. Mott, T. M. Whittle, "Well test analysis in gas-condensate reservoirs", SPE Annual Technical Conference and Exhibition, Dallas, USA, October 1-4, 2000

[10] M. S. Abdallah, M. A. Al-Zawad, M. L. Fraim, "Common misinterpretations of gas condensate reservoirs", SPE Kingdom of Saudi Arabia Annual Technical Symposium and Exhibition, Dammam, Saudi Arabia, April 24-27, 2017

[11] M. H. Chachar, S. A. Jokhio, A. H. Tunio, "Investigating gas phase productivity in gas condensate reservoir: a review", Engineering Science \& Technology International Research Journal, Vol. 2, No. 3, pp. 95-99, 2018

[12] S. A. Jokhio, D. Tiab, S. Baladi, A. H. Tunio, "Estimating pseudopressure function without relative permeability $[\mathrm{kr}(\mathrm{Sw})]$ for two and three phase gas condensate systems", SPE Asia Pacific Oil and Gas Conference and Exhibition, Melbourne, Australia, October 8-10, 2002

[13] R. Al-Hussainy, H. J. Ramey, P. B. Crawford, "The flow of real gases through porous media", Journal of Petroleum Technology, Vol. 18, No. 5, pp. 624-636, 1966

[14] P. Gherson, C. Faruk, "Gas-condensate well test analysis with and without relative permeability curves", SPE Annual Technical Conference and Exhibition, Dallas, USA, October 1-4, 2000

[15] V. N. Gopal, "Gas Z-factor equations developed for computer", Oil and Gas Journal, Oil and Gas Journal, Vol. 75, No. 8, pp. 8-13, 1977

[16] A. L. Lee, M. H. Gonzalez, B. E. Eakin, "The viscosity of natural gases", Journal of Petroleum Technology, Vol. 18, No. 8, pp. 997-1001, 1966 